\title{
Sum-Capacity of Massive MIMO Systems Using Vandermonde Matrices
}

\author{
Claudio F. Dias, Michelle S. P. Facina, Felipe A. P. de Figueiredo, Eduardo R. Lima, and Gustavo
}

Fraidenraich

\begin{abstract}
In this paper, we use a series expansion to calculate the sum-capacity of a massive Multiple-Input MultipleOutput (MIMO) system under propagation environment described by a dominant line-of-sight. The sum-capacity is written as Taylor's series where each term is a function of the mean trace of $k$-th power of the channel matrix W. We analytically derive the mean trace of first, second, third, and fourth moments of $W$. Although the series is infinite, our numerical results show that only a few terms can tightly approximate the exact sum capacity. Numerical results corroborate our analytical expressions.
\end{abstract}

Index Terms-Massive MIMO, Line-of-sight, Sumcapacity, Vandermonde Matrix.

\section{INTRODUCTION}

The industry and academia are struggling to give solutions that can support various emerging applications for smart devices such as ultra-high definition video streaming, 3D virtual reality, and video conferencing that have triggered unforeseen demand for higher data rates. The massive Multiple-Input Multiple-Output (MIMO) has been proposed as a promising technique and has attracted growing interests mainly because i) it provides the possibility to vanish the uncorrelated noise effects and fast fading; ii) it grants the throughput and the number of terminals to be independent of the cell size; iii) it allows the spectral efficiency to be independent of bandwidth, and; iv) it reduces the required transmitted energy per bit [1].

A good ferature is that when applied at high frequencies and taking advantage of the vast amount of frequencies available in the range of 3 to $300 \mathrm{GHz}$ [2], [3], massive MIMO combines with the millimeter wave technology and the classical Line-of-Sight (LoS) propagation becomes more dominant as a consequence of the higher radiation absorption and increased path loss [4]. Although its efficiency in a rich scattering environment has already been demonstrated, less is known about its performance under this new scenario.

In the pioneering work [5], Telatar has found the pointto-point average MIMO capacity and was able to analytically derive the joint eigenvalue density for a Wishart matrix. However for massive MIMO, the metric of interest is the uplink sum-capacity that can measure the maximum throughput inside a cell composed by a base station and many mobile units. In this case, when the channel between each cell and the base station is modeled as pure LoS, the corresponding channel matrix $\boldsymbol{H}$ boils down to a Vandermonde matrix [6]. Unfortunately, the joint

C. F. Dias, M. S. P. Facina, Felipe A. P. de Figueiredo and, Eduardo R. Lima and, G. Fraidenraich are with Dept. of Electrical Engineering, University of Campinas, Brazil, e-mail: \{aplnx0xff, michelle.facina, zz4fap, gustavofraidenra\}@gmail.com. eigenvalue density function for this kind of structure is unknown and alternative methods have to be developed to compute the sum-capacity.

In many research fields, mainly in digital communications, channel like this can be modeled through random matrices. When these matrices get large, free probability theory is an invaluable tool for describing the asymptotic behavior of systems [7].

From a mathematical point of view, Tucci et. al. obtain capacity bounds of Vandermonde channel through empirical probability densities of eigenvalues [8].

In this Letter, we present an accurate expression to calculate the capacity of massive multiple-input multipleoutput systems under the assumption that the propagation environment is likely to be better described as a dominant LoS. Our approach expands the sum-capacity as Taylor's series where each term is a function of the trace of powers of the channel matrix $\mathbf{W}$.

The mathematical proofs that underlie our approach are best detailed in [9]. The validity of our approximation is extended not only to uniform user distribution but also when the users are concentrated in a region cell. To the best of author's knowledge, no similar contributions have been found in the literature.

The remaining of this paper is organized as follows: Section II refers to the system model while Section III presents the analytical expressions for massive MIMO capacity. Section IV shows numerical simulations that confirm the accuracy of approximation. Finally, we close our discussion in the Section V summarizing our conclusions.

\section{A. Notation}

Scalars are denoted by lower-case letters, vectors by bold-face lower-case letters, and matrices by bold-face upper-case letters. The identity matrix and the all-zero matrix of appropriate dimensions are denoted as $\mathbf{I}$ and $\mathbf{0}$, respectively. For a matrix $\mathbf{G}$ of arbitrary size, $G^{H}$ and $G^{T}$ denote its conjugate transpose and transpose, respectively. The expectation operator is denoted as $\mathbb{E}$. The distribution of a circularly symmetric complex Gaussian (CSCG) random vector with mean $x$ and covariance matrix $\Sigma$ is denoted by $\mathcal{C N}(x, \Sigma)$.

\section{MIMO SYSTEM MODEL}

Consider the uplink of a single cellular network occupied by $N$ users. It is assumed that the BSs hold a determined number of antennas, $M$. For operation simplicity, the Orthogonal Frequency Division Multiplexing (OFDM)/Time Division Duplex (TDD) is used. In this way, the channel becomes flat and exhibits a reciprocity 
behavior, reducing the overhead required for the acquisition of Channel State Information (CSI) by means of uplink training signals.

Then, the channel input-output relationship for the uplink communication can be given as

$$
\mathbf{y}=\mathbf{H x}+\mathbf{n},
$$

where $\mathbf{y} \in \mathbb{C}^{M \times 1}$ and $\mathbf{x} \in \mathbb{C}^{N \times 1}$ are, respectively, the user received and transmitted signals. $\mathbf{H}$ is an $M \times N$ matrix that represents the frequency response of the channel between base station and users while $\mathbf{n} \in \mathbb{C}^{M \times 1}$ is the additive white Gaussian noise (AWGN) vector distributed as $C N\left(\mathbf{0}, \sigma^{2} \mathbf{I}\right)$.

Under LOS propagation and considering an uniform linear array (ULA) whose distance between the base station antennas is $d_{\lambda}=\frac{\lambda}{2}$, we can model the MIMO channel $\mathbf{H}$ as the following Vandermonde structure, with unit magnitude complex entries

$$
\begin{aligned}
\mathbf{H} & =\left[\begin{array}{llll}
\mathbf{1} & e^{-i 2 \pi \frac{d_{\lambda}}{\lambda} \boldsymbol{\theta}} & \cdots & e^{-i(M-1) 2 \pi \frac{d_{\lambda}}{\lambda} \boldsymbol{\theta}}
\end{array}\right]^{T} \\
& =\frac{1}{\sqrt{M N}}\left[\begin{array}{ccc}
1 & \cdots & 1 \\
\mathrm{e}^{-i \pi \theta_{1}} & \cdots & \mathrm{e}^{-i \pi \theta_{N}} \\
\mathrm{e}^{-i 2 \pi \theta_{1}} & \cdots & \mathrm{e}^{-i 2 \pi \theta_{N}} \\
\vdots & \ddots & \vdots \\
\mathrm{e}^{-i(M-1) \pi \theta_{1}} & \cdots & \mathrm{e}^{-i(M-1) \pi \theta_{N}}
\end{array}\right],
\end{aligned}
$$

where $i=\sqrt{-1}$ is the complex symbol and $\boldsymbol{\theta}=$ $\left[\begin{array}{lll}\theta_{1} & \theta_{2} \ldots \theta_{N}\end{array}\right]^{T}$ is a column vector whose $n$-th element is an independent and identically distributed (i.i.d.) random variable and can be written by

$$
\theta_{n}=\left\{\begin{array}{cc}
\alpha_{n}, & \alpha_{n} \in[-1,1] \\
\sin \left(\alpha_{n}\right), & \alpha_{n} \in[-\pi, \pi]
\end{array},\right.
$$

in which $\alpha_{n}$ is the angle of the $n$-th user with respect to the base station. It can also be shown that we can distribute the users uniformly in area and find an equivalent fixed radius that represent the system.

Assuming that all users transmit at constant unitary power, the capacity of a MIMO system described by (1) becomes, [10]

$$
\begin{aligned}
C & =\log _{2}\left|I_{M}+\rho \mathbf{H} \mathbf{H}^{H}\right| \\
& =\log _{2}\left|I_{N}+\rho \mathbf{H}^{H} \mathbf{H}\right| .
\end{aligned}
$$

where $\rho$ is the average signal-to-interference ratio (SNR).

In the remainder of this paper, $\mathbf{H}$ is taken to be normalized channel matrix, which implies that each element of $\mathrm{H}$ has unit average power. By requiring this normalization we make the average SNR independent of $\mathbf{H}$. The eigenvalue decomposition of $\mathbf{H}$ in (4) leads to the following alternative representation of the system capacity

$$
C \approx \sum_{l=1}^{N} \mathbb{E}\left[\log _{2}\left(1+\rho \lambda_{l}(\boldsymbol{\theta})\right)\right] \text { bits/s/Hz, }
$$

where $\lambda_{l}(\boldsymbol{\theta})$ is the $l$-th eigenvalue of $\mathbf{W}$, defined as [5]

$$
\mathbf{W}= \begin{cases}\mathbf{H}^{H} \mathbf{H}, & M>N \\ \mathbf{H H}^{H}, & M \leq N .\end{cases}
$$

Note that we have explicitly used $\lambda_{l}(\boldsymbol{\theta})$ to clearly show the dependency of the eigenvalues with respect to the distribution of the users angles. Thus, the mean capacity can also be represented a

$$
C=\sum_{l=1}^{N} \int \log _{2}\left(1+\rho \lambda_{l}\right) f(\boldsymbol{\theta}) d \boldsymbol{\theta},
$$

where $f(\boldsymbol{\theta})$ is the joint probability density function (PDF) of the random vector $\boldsymbol{\theta}$. Considering that all elements of $\boldsymbol{\theta}$ are independent and uniformly distributed between 0 and $2 \pi$, then $f(\boldsymbol{\theta})=\prod_{i=1}^{N} f\left(\theta_{i}\right)=\left(\frac{1}{2 \pi}\right)^{N}$.

\section{CAPACITY AND MOMENTS}

We can expand the term $\log _{2}\left(1+\rho \lambda_{l}\right)$ in the eq. (7) using the Taylor series [9] and then rewrite it as

$$
C=\sum_{l=1}^{N} \frac{1}{\ln (2)} \sum_{k=1}^{\infty} \frac{(-1)^{k+1} \rho^{k} \lambda_{l}^{k} \int f(\boldsymbol{\theta}) d \boldsymbol{\theta}}{k} .
$$

Since $\mathbf{W}$ is a symmetric matrix, the trace operator is equal to the sum of its eigenvalues, ie., $\operatorname{Tr}[\mathbf{W}]=\sum_{t=1}^{N} \lambda_{t}$ [6]. In the same way, there is the averaged trace $\mathbb{E}[\operatorname{Tr}()$. for $N \times N$ random matrices whose elements are random variables on some probability space $\Omega$ defined as

$$
\mathbb{E}[\operatorname{Tr}(\mathbf{W})]=\sum_{l=1}^{N} \mathbb{E}\left[\lambda_{l}\right]=\sum_{l=1}^{N} \int_{\Omega} \lambda_{l} f(\boldsymbol{\theta}) d \boldsymbol{\theta},
$$

Therefore, we can rewrite (8) as

$$
C=\frac{1}{\ln (2)} \sum_{k=1}^{\infty} \frac{(-1)^{k+1} \rho^{k}}{k} \mathbb{E}\left[\operatorname{Tr}\left(\mathbf{W}^{k}\right)\right] .
$$

From (10), it is possible to compute the mean sum capacity using the mean of the trace of the moments of $\boldsymbol{W}$.

\section{A. Derivation of $\operatorname{Tr}\left[\mathbf{W}^{k}\right]$}

The analytical expression for capacity is deduced from the term $\mathbb{E}\left[\operatorname{Tr}\left(\mathbf{W}^{k}\right)\right]$ for $k$-th moment. Then, lets call each term of matrix $\mathbf{W}$ as $w_{i j}$, in which the indexes $i$ and $j$ refer to users, and $\theta_{i j}$ is the difference between the angle $\theta_{n}$ of two of them, i.e. $\theta_{i j}=\theta_{i}-\theta_{j}$. So,

$$
w_{i j}=\sum_{m=0}^{(M-1)} \mathrm{e}^{i m \pi \theta_{i j}} .
$$

Note that since the terms $w_{11}=w_{22}=\ldots=w_{N N}=1$, we can write

$$
\begin{gathered}
\operatorname{Tr}(\mathbf{W})=\sum_{i=1}^{N} w_{i i}=1 \\
\operatorname{Tr}\left(\mathbf{W}^{2}\right)=\sum_{i=1}^{N} \sum_{j=1}^{N} w_{i j} w_{i j} \\
=\frac{1}{M N}+2 \sum_{i=1}^{N} \sum_{i>j}^{N} w_{i j} w_{i j}^{\dagger},
\end{gathered}
$$

where $(\cdot)^{\dagger}$ is the conjugate operator. Since the distributive product of $w_{i j} w_{i j}^{\dagger}$ produces

$$
\begin{aligned}
w_{i j} w_{i j}^{\dagger}= & 1+\mathrm{e}^{-i \pi \theta_{i j}}+\ldots+\mathrm{e}^{-i(M-1) \pi \theta_{i j}}+ \\
& \ldots+\mathrm{e}^{i \pi \theta_{i j}}+1+\mathrm{e}^{-i \pi \theta_{i j}}+\ldots \\
& +\mathrm{e}^{i(M-1) \pi \theta_{i j}}+\ldots+\mathrm{e}^{-i \pi \theta_{i j}}+1,
\end{aligned}
$$


that has elements with conjugate symmetry, the difference between imaginary symmetric terms vanishes and only real cosine terms remain. Thus,

$$
w_{i j} w_{i j}^{\dagger}=\frac{1}{M N}\left(M+2 \sum_{m=1}^{(M-1)}(M-m) \cos \left(m \pi \theta_{i j}\right)\right) \text {. }
$$

Finally, (13) can be expressed as

$$
\operatorname{Tr}\left(\mathbf{W}^{2}\right)=\frac{1}{M N}+\frac{4}{M N} \sum_{i_{1}=1}^{N} \sum_{i_{2}>i_{1}}^{N} \sum_{m=0}^{(M-1)}(M-m) \cos \left(m \pi \theta_{i j}\right) .
$$

On the other hand, the trace of $\mathbf{W}^{3}$ is

$$
\operatorname{Tr}\left(\mathbf{W}^{3}\right)=\sum_{i=1}^{N} \sum_{j=1}^{N} w_{i j} \sum_{l=1}^{N} w_{l i} w_{j l} .
$$

and it can be separated into the following summation terms

$$
\begin{aligned}
\operatorname{Tr}\left(\mathbf{W}^{3}\right) & =\sum_{i=1}^{N} w_{i i}^{3} \\
& +\sum_{i=1}^{N} w_{i i} \sum_{l \neq i}^{N} w_{l i} w_{i l} \\
& +\sum_{i=1}^{N} \sum_{j \neq i}^{N} w_{i j} \sum_{l=i}^{N} w_{l i} w_{j l} \\
& +\sum_{i=1}^{N} \sum_{j \neq i}^{N} w_{i j} \sum_{l=j}^{N} w_{l i} w_{j l} \\
& +\sum_{i=1}^{N} \sum_{j \neq i}^{N} w_{i j} \sum_{l \neq i, l \neq j}^{N} w_{l i} w_{j l},
\end{aligned}
$$

in which the second and third ones resemble to the difference between imaginary symetric terms. In addition, since $\mathbf{W}$ is hermitian, we can perform further simplifications and (18) becomes

$$
\begin{aligned}
\operatorname{Tr}\left(\mathbf{W}^{3}\right)= & \frac{1}{(M N)^{2}} \\
& +6 \sum_{i=1}^{N} \sum_{j>i}^{N} w_{i j} \sum_{l=i}^{N} w_{l i} w_{j l} \\
& +\sum_{i=1}^{N} \sum_{j \neq i}^{N} w_{i j} \sum_{l \neq i, l \neq j}^{N} w_{l i} w_{j l} .
\end{aligned}
$$

It is worth mentioning that we have tried to extend our results to higher orders. However, the mathematical complexity is prohibitive as the number of terms in the expansion of $\operatorname{Tr}\left[\boldsymbol{W}^{k}\right]$ ( $k \geq 4$ grows exponentially. Fortunately, as it will be shown in the numerical results section, for a high number of antennas the first three terms of the series provide a high accuracy to the exact result.

\section{B. Computation of $\mathbb{E}\left[\operatorname{Tr}\left(\mathbf{W}^{k}\right)\right]$}

The calculation of (10) requires defining the $k$-moment from two possible PDFs for the random variable $\alpha_{n}$.
1) $\alpha_{n} \sim \mathcal{U}[-1,1]$ and $\theta_{n}=\alpha_{n}$ : As $\alpha_{n}$ is uniformly distributed, the PDF of $\theta_{n}$ is $f\left(\theta_{n}\right)=1 / 2$ with $\theta_{n} \in$ $[-1,1]$. The first moment is directly derived as $m_{1}=1$. In (16), the summation terms integrates to zero except for $m=0$. Then,

$$
\begin{aligned}
m_{2} & =\mathbb{E}\left[\operatorname{Tr}\left(\mathbf{W}^{2}\right)\right] \\
& =\frac{1}{(M N)^{2}}\left(M^{2} N+M \frac{N !}{(N-2) !}\right)
\end{aligned}
$$

as it also happens in (19) that results in

$$
\begin{aligned}
m_{3} & =\mathbb{E}\left[\operatorname{Tr}\left(\mathbf{W}^{3}\right)\right] \\
& =\frac{1}{(M N)^{3}}\left(M^{3} N+3 M^{2} \frac{N !}{(N-2) !}+M \frac{N !}{(N-3) !}\right) .
\end{aligned}
$$

In its turn, we have that

$$
\begin{aligned}
m_{4} & =\mathbb{E}\left[\operatorname{Tr}\left(\mathbf{W}^{4}\right)\right] \\
& =\frac{1}{(M N)^{4}}\left(M^{4} N+\frac{\left(M+20 M^{3}\right) N !}{3(N-2) !}\right. \\
& \left.+6 M^{2} \frac{N !}{(N-3) !}+M\right) .
\end{aligned}
$$

2) $\alpha_{n} \sim \mathcal{U}[-\pi, \pi]$ and $\theta_{n}=\sin \left(\alpha_{n}\right)$ : Again, $\alpha_{n}$ is uniformly distributed, but due to variable transformation, the PDF of $\theta_{n}$ is $f\left(\theta_{n}\right)=\frac{1}{\pi \sqrt{1-\theta_{n}^{2}}}$ and $\theta_{n} \in[-1,1]$. Then, the first moment is $m_{1}=1$ while the second one is given by

$$
\begin{aligned}
m_{2} & =\frac{1}{(M N)^{2}}\left(M^{2} N\right. \\
& +M \frac{N !}{(N-2) !} \\
& \left.+\sum_{m=1}^{M} 2(M-m) J_{0}(m \pi)^{2} \frac{N !}{(N-2) !}\right),
\end{aligned}
$$

where $J_{0}(\cdot)$ is the Bessel function of the first kind.

Similarly, the third moment can be written as

$$
\begin{aligned}
m_{3} & =\frac{1}{(M N)^{3}}\left(M^{3} N+3 M^{2} \frac{N !}{(N-2) !}+M \frac{N !}{(N-3) !}\right. \\
& +\sum_{m=1}^{(M-1)} 6 M(M-m) J_{0}(m \pi)^{2} \frac{N !}{(N-2) !} \\
& +\sum_{m=1}^{(M-1)} 6(N-2) \prod_{k=0}^{2}(m+k) J_{0}((M-m) \pi)^{2} \\
& +\sum_{m=1}^{\frac{M-1}{2}} 6(N-2) \prod_{k=0}^{2}(M-2 m+k) J_{0}(m \pi)^{2} J_{0}(2 m \pi) \\
& +\sum_{m=1}^{(M-1)} \sum_{l=1}^{M-2 m} u(M-2 m) 6(N-2) \\
& \times \prod_{k=0}^{2}(2(M-l-1-2(m-1))+k) \\
& \left.\times J_{0}(m \pi) J_{0}((m+l) \pi) J_{0}((2 m+l) \pi)\right)
\end{aligned}
$$

where $u(\cdot)$ is a step function. 


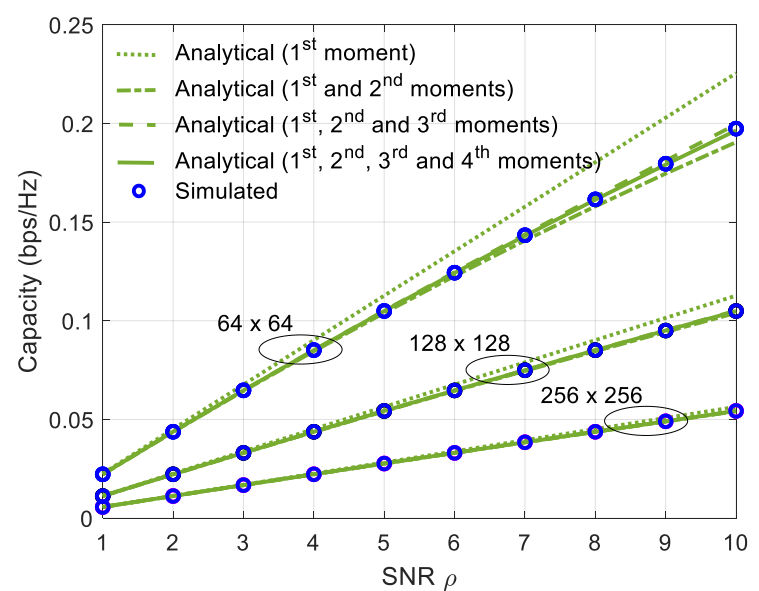

Fig. 1. Comparison between the analytical and simulated capacity when $M=N$ and $f\left(\theta_{n}\right)=\frac{1}{2}$.

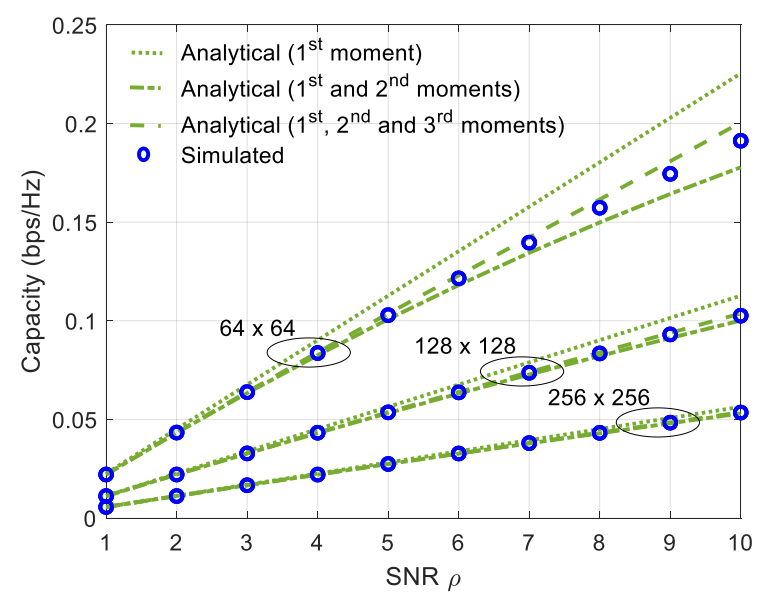

Fig. 2. Comparison between the analytical and simulated capacity when $M=N$ and $f(\boldsymbol{\theta})=\frac{1}{\pi \sqrt{1-\theta_{n}^{2}}}$.

\section{NUMERICAL RESUlTS}

In this section, we present the numerical results comparing the approximation form of the capacity and simulations for the two distributions described before. As many applications involve large quantity of $\mathrm{M}$ and $\mathrm{N}$, we have performed simulations taking into account $M=N$ and $M$ ranging from 64 to 256 antennas. For the plots, $10^{4}$ samples were used to calculate the statistics.

Fig. 1 shows the analytical and simulated results, assuming $f\left(\theta_{n}\right)=\frac{1}{2}$ and the dimension of $\mathbf{H}$ as $M \times N$. The blue color is used for simulations while the green color refers to the analytical results. In this case, the green curves are obtained considering the approximated capacity by until four moments at (10). It is evident the accuracy of the presented method. For this PDF, the analytical results are very close to the simulated ones, even for matrices of reduced dimensions.

Similarly, Fig. 2 presents the analytical capacity calculated through (10). Considering now $f\left(\theta_{n}\right)=\frac{1}{\pi \sqrt{1-\theta_{n}^{2}}}$, it is clear that the approximation fits well as long as the number of antennas and $k$ increases.

\section{CONCLUSION}

In this work, we investigated how the user arrangement can influence the sum capacity of a massive MIMO system under dominant LoS. We derived analytical expressions for capacity that depend only on the parameters $M$ (number of antennas), and $N$ (number of users). The results were validated by numerical simulations and showed an excellent agreement.

As future work, we intend to extend the approximation also to uniform rectangular array.

\section{ACKNOWLEDGMENT}

The authors would like to thank the grant \#2016/161812, São Paulo Research Foundation (FAPESP), for the support in this research.

\section{REFERENCES}

[1] T. L. Marzetta, "Noncooperative cellular wireless with unlimited numbers of base station antennas," IEEE Transactions on Wireless Communications, vol. 9, no. 11, pp. 3590-3600, 2010.

[2] Z. Pi and F. Khan, "A millimeter-wave massive mimo system for next generation mobile broadband," in Conference on Signals, Systems and Computers (ASILOMAR). IEEE, 2012, pp. 693-698.

[3] M. Jaber, M. A. Imran, R. Tafazolli, and A. Tukmanov, "5G backhaul challenges and emerging research directions: A survey," IEEE Access, vol. 4, pp. 1743-1766, 2016.

[4] Z. Pi and F. Khan, "An introduction to millimeter-wave mobile broadband systems," IEEE Communications Magazine, vol. 49, no. 6, pp. 101-107, Jun. 2011.

[5] E. Telatar, "Capacity of multi-antenna gaussian channels," Transactions on Emerging Telecommunications Technologies, vol. 10, no. 6, pp. 585-595, 1999.

[6] R. A. Horn and C. R. Johnson, Matrix Analysis, 2nd ed. New York, NY, USA: Cambridge University Press, 2012.

[7] O. Ryan and M. Debbah, "Signal processing applications of free probability theory," in European Signal Processing Conference, Sep. 2007, pp. 1181-1185.

[8] G. H. Tucci and P. A. Whiting, "Eigenvalue results for large scale random vandermonde matrices with unit complex entries," IEEE Transactions on Information Theory, vol. 57, no. 6, pp. 3938-3954, Jun. 2011.

[9] O. Ryan and M. Debbah, "Asymptotic behavior of random vandermonde matrices with entries on the unit circle," IEEE Transactions on Information Theory, vol. 55, no. 7, pp. 3115-3147, Jul. 2009.

[10] N. H. Q. Larsson, E. G. and H. Yang, Fundamentals of Massive $M I M O, 1$ st ed. New York, NY, USA: Cambridge University Press, 2016. 